\title{
Interference-Based Capacity Analysis for Vehicular Ad Hoc Networks
}

\author{
Minming Ni, Jianping Pan, Lin Cai, Jian Yu, Hao Wu, and Zhangdui Zhong
}

\begin{abstract}
Although some scaling law-based studies have been conducted for describing the asymptotic capacity of Vehicular Ad Hoc Networks (VANETs), the obtained results are not easy to directly use for estimating the actual capacity of a communication link or the entire network. To overcome this drawback, an interference-based capacity analysis is carried out in this letter for the abstracted 1-dimensional VANETs scenario. For a reasonable and tractable modeling, the classic Car-Following model is applied to represent the dynamic change of the inter-vehicle distance, which strongly affects the power decay of both the signal and interference in the network. Based on that, a series of probability characteristics is derived for the worst-case interfering scenario, which finally leads to the stochastic characteristics for both the link and network capacity.
\end{abstract}

Index Terms-VANETs, interference, probability analysis, capacity.

\section{INTRODUCTION}

$\mathbf{I}$ $\mathrm{N}$ view of the potential for improving the safety, efficiency, and effectiveness of the current transportation system, the Vehicular Ad Hoc Networks (VANETs) have attracted much attention from both industry and academia. However, despite the increasing amount of research on VANETs, the characteristics of VANETs capacity are still not very clear. Generally, most of the exiting studies for VANETs capacity (e.g., [1], [2]) still follow the classic scaling-law based analysis method. However, since these results only describe how per-node capacity scales in an asymptotically large network, they are not easy to be used for estimating or evaluating the actual performance of a link or the entire network when a specific group of parameter settings is given.

In view of this drawback, an interference-based capacity analysis is carried out in this letter specifically for the VANETs scenario. For incorporating the impact of vehicle mobility on the transmission performance, the Car-Following model is utilized in our analyses to obtain the stochastic characteristics for both the signal's and interference's propagation distance of a tagged communication pair. Based on that, the probability density function (PDF) of the Signal-to-Interference Ratio

Manuscript received August 23, 2014; revised January 8, 2015; accepted January 9, 2015. Date of publication January 14, 2015; date of current version April 8, 2015. This work is partly supported by the NSFC (Grant No. 61401016), State Key Laboratory of Rail Traffic Control and Safety (Grant No. RCS2014ZT33, RCS2014ZQ003, and RCS2012ZT007), the Fundamental Research Funds for the Central Universities (Grant No. 2014JBM154), NSERC (with contributions from DRDC), CFI, and BCKDF. The associate editor coordinating the review of this paper and approving it for publication was A. Vinel.

M. Ni and $\mathrm{H}$. Wu are with the State Key Laboratory of Rail Traffic Control and Safety, Beijing Jiaotong University, Beijing 100044, China (e-mail: mmni@bjtu.edu.cn; hwu@bjtu.edu.cn).

J. Pan is with the Department of Computer Science, University of Victoria, Victoria, BC V8W 2Y2, Canada (e-mail: pan@uvic.ca).

L. Cai is with the Department of Electrical and Computer Engineering, University of Victoria, Victoria, BC V8W 2Y2, Canada (e-mail: cai@ece.uvic.ca).

J. Yu and Z. Zhong are with the School of Computer and Information Technology, Beijing Jiaotong University, Beijing 100044, China (e-mail: jianyu@ bjtu.edu.cn; zhdzhong@bjtu.edu.cn).

Digital Object Identifier 10.1109/LCOMM.2015.2392117
(SIR) at the observed receiver can then be obtained, which finally leads to the expected link and network capacity. The most relevant work in the literature is [3], which conducted an interference-based performance analysis with the hidden and exposed terminal problem. However, the constant-speed mobility model was used in [3], which may not able to fully demonstrate the dynamic constraint on the distance between neighboring vehicles.

The contributions of this letter are twofold. First, the distribution of the interference's propagation distance is derived for a generalized worst-case interfering scenario, which could benefit many other related research issues. Second, with the analysis method proposed in this letter, the communication quality of a randomly selected receiver can be presented as the PDF of its SIR, which is much easier to be applied for the design of networking or transmission schemes for VANETs.

\section{SYSTEM MODEL}

According to the IEEE $802.11 \mathrm{p}$ protocol, the common transmission range of a vehicle varies between 500 and 1000 meters depending on the different network settings. Therefore, it is reasonable to abstract the actual 2-D strip-like network area to a 1-D single lane, which is useful for describing the network topology and easy to be applied for theoretical analyses. Besides, we also assume that all the vehicles are moving along the abstracted linear road with the classic Car-Following model [4], which means that the distance $D$ between any two neighboring vehicles follows a log-normal distribution with parameters $\mu$ and $\sigma$.

For simplicity, all the network nodes (vehicles on the road) are treated as homogeneous, which means that they have identical vehicle length $L_{\mathrm{v}}$ and transmission power $P_{\mathrm{t}}$. To demonstrate the impact of the wireless channel on the capacity while keeping the analysis tractable, the Nakagami fading model is applied to describe the channel's fading effect, which is easy to be degenerated to more specific propagation scenarios. Therefore, the power $P_{\mathrm{r}}$ received from an interferer with distance $d$ away has the PDF as

$$
f_{P_{\mathrm{r}} \mid d}(x)=\frac{1}{\Gamma(m)}\left(\frac{m}{\bar{P}_{\mathrm{r}}(d)}\right)^{m} x^{m-1} \exp \left(-\frac{m x}{\bar{P}_{\mathrm{r}}(d)}\right),
$$

where $\Gamma(\cdot)$ is the Gamma function, and $m$ is the fading parameter. Besides, $\bar{P}_{\mathrm{r}}(d)$ is the mean value determined by the pathloss as $\bar{P}_{\mathrm{r}}(d)=P_{\mathrm{t}} \beta\left(d_{0} / d\right)^{\alpha}$, where $\beta$ is a transceiver-determined constant, $d_{0}$ is the reference distance for the far-zone field, and $\alpha$ is the pathloss exponent. Based on the homogeneous assumption, $\alpha, \beta$, and $m$ are identical for all the coexisting data transmissions, respectively.

For the media access control, the standard CSMA/CA scheme is applied as designated in the IEEE $802.11 \mathrm{p}$ protocol. Therefore, for a tagged transmitter $i$, the channel sensing procedure before initiating its transmission creates an exclusive region (ER) [5] centered at $i$ with radius $r_{\mathrm{E}}=d_{0} \sqrt[\alpha]{P_{\mathrm{t}} \beta / P_{\mathrm{th}}}$, where $P_{\text {th }}$ is the clear channel assessment (CCA) sensitivity. As a result of the channel sensing mechanism, there will be 


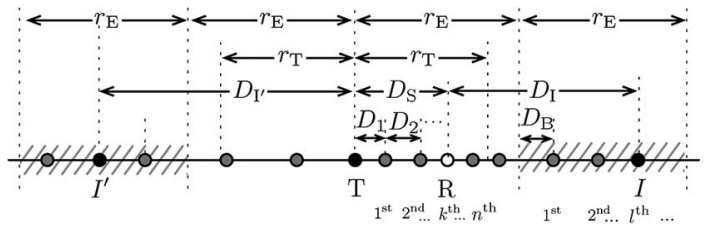

Fig. 1. A general interfering scenario for VANETs.

only one active transmitter within each ER [6]. ${ }^{1}$ Hence, the interferers for a tagged receiver $\mathrm{R}$ should at least stay with distance $r_{\mathrm{E}}$ away from R's transmitter T. Moreover, due to the power attenuation along with the signal propagation, the part of the interferers located close to the tagged communicating pair will generate the majority of the interference aggregated at $\mathrm{R}$. Hence, we can only consider the impact of the closest interferer on T's both sides, which has already been confirmed to be accurate enough in [7]. To obtain the capacity when the network is densely deployed (e.g., when the traffic jam happens), which could be considered as the worst-case scenario for a tagged communication pair, the distance between an interferer and the tagged transmitter $\mathrm{T}$ should also be no longer than $2 r_{\mathrm{E}}$. Besides, a ratio parameter $\gamma$ is also defined for representing the difference between the sensing range $r_{\mathrm{E}}$ and the expected transmission range $r_{\mathrm{T}}$, which is determined by the minimum reception SIR requirement, as $\gamma=r_{\mathrm{T}} / r_{\mathrm{E}}$. Normally, $\gamma$ is set to 0.5 , but it can always be tuned to other values for capturing more specific receiver hardware features. By combining all the above assumptions, the two interferers for receiver $\mathrm{R}$ should be located within the two shaded regions depicted in Fig. 1, respectively. As shown in the figure, the black dots are the active transmitters, the white dot is the tagged receiver, and the gray dots are the network nodes suppressed by the CSMA scheme.

\section{THEORETICAL ANALYSIS}

\section{A. Signal Propagation Distance}

According to the system model, given that the observed receiver $\mathrm{R}$ is the $k$-th node within the transmitter T's right-side transmission range, ${ }^{2}$ the signal's propagation distance $D_{\mathrm{S}}$ can be presented as $\sum_{i=1}^{k} D_{i}$, where $D_{1}$ is the distance between T and its first right-side neighbor, and $D_{i}(i \in[2, k])$ represents the distance between the $(i-1)$-th and $i$-th neighbors on T's right side. For easy description, we use notation $Y_{M}$ to represent the sum of $M$ neighboring vehicle inter-distance $(M \geq 1)$. According to the Fenton-Wilkinson (FW) method [8], $Y_{M}$ 's PDF $f_{Y_{M}}(x)$ can be approximated by another log-normal distribution as $\frac{1}{\sqrt{2 \pi} \sigma_{M} x} \exp \left(\frac{-\left(\ln x-\mu_{M}\right)^{2}}{2 \sigma_{M}^{2}}\right)$, where $x>0, \sigma_{M}=\ln \left(\left(e^{\sigma^{2}}-1\right) / M+1\right)$, $\mu_{M}=\ln \left(M e^{\mu}\right)+\left(\sigma^{2}-\sigma_{M}^{2}\right) / 2$. Considering that $D_{\mathrm{S}} \in\left(0, r_{\mathrm{T}}\right]$, when $k$ is given, the conditional PDF of $D_{\mathrm{S}}$ needs to be normalized as $f_{D_{\mathrm{S}} \mid k}(x)=f_{Y_{k}}(x) / \int_{0}^{r_{\mathrm{T}}} f_{Y_{k}}(x) \mathrm{d} x$. With the total probability formula, the unconditional PDF of $D_{\mathrm{S}}$ can be presented as

$$
f_{D_{\mathrm{S}}}(x)=\sum_{k=1}^{\left\lfloor r_{\mathrm{T}} / L_{\mathrm{V}}\right\rfloor} f_{Y_{k}}(x) \cdot \operatorname{Pr}\{\mathrm{R} \text { is the } k \text {-th node }\} .
$$

Based on the assumption of the average vehicle length $L_{\mathrm{V}}, k$ should be upper bounded to $\left\lfloor r_{\mathrm{T}} / L_{\mathrm{V}}\right\rfloor$ rather than go to infinity as

\footnotetext{
${ }^{1}$ Here, we ignore the collision probability for simplicity. As the collision probability in the MAC layer has been extensively investigated in the literature, it is ready to further extend this work considering the collisions.

${ }^{2}$ When $\mathrm{R}$ is located on the left side of $\mathrm{T}$, similar results can be obtained with the identical method proposed in this letter.
}

shown in (2). Meanwhile, if we use $N$ to represent the number of nodes within T's right-side transmission range, the probability for $\mathrm{R}$ to be the $k$-th node on T's right side can be decomposed as

$$
\begin{aligned}
\operatorname{Pr}\left\{\mathrm{R} \text { is the } k \text {-th node in } \mathrm{T}^{\prime} \text { s right-side TX range }\right\} \\
=\sum_{n=1}^{\left\lfloor r_{\mathrm{T}} / L_{\mathrm{v}}\right\rfloor} \operatorname{Pr}\{N=n\} \operatorname{Pr}\{\mathrm{R} \text { is the } k \text {-th node } \mid N=n\} \\
=\sum_{n=1}^{\left\lfloor r_{\mathrm{T}} / L_{\mathrm{v}}\right\rfloor} \underbrace{\operatorname{Pr}\left\{Y_{n} \leq r_{\mathrm{T}}\right\} \operatorname{Pr}\left\{Y_{n}+D_{n+1}>r_{\mathrm{T}} \mid Y_{n} \leq r_{\mathrm{T}}\right\}}_{\text {Part } A} \\
\quad \times \underbrace{\operatorname{Pr}\{\mathrm{R} \text { is the } k \text {-th node } \mid N=n\}}_{\text {Part } B} .
\end{aligned}
$$

Based on the independency between the two random variables $Y_{n}$ and $D_{n+1}$, Part $A$ in (3) can be calculated as

$$
\begin{aligned}
\operatorname{Pr}\left\{Y_{n}\right. & \left.\leq r_{\mathrm{T}}\right\} \operatorname{Pr}\left\{Y_{n}+D_{n+1}>r_{\mathrm{T}} \mid Y_{n} \leq r_{\mathrm{T}}\right\} \\
& =\Phi\left(\frac{\ln r_{\mathrm{T}}-\mu_{n}}{\sigma_{n}}\right)-\int_{0}^{r_{\mathrm{T}}} \Phi\left(\frac{\ln \left(r_{\mathrm{T}}-x\right)-\mu}{\sigma}\right) f_{Y_{n}}(x) \mathrm{d} x
\end{aligned}
$$

where $\Phi(\cdot)$ is the cumulative distribution function (CDF) of the standard normal distribution, and both the two parameters $\mu_{n}$ and $\sigma_{n}$ should also be adjusted according to the FW method. For Part $B$ in (3), different transmission schemes may lead to different results. In this letter, we only consider a general case that, the selection of a receiver can be treated as uniform in a long-term view. For example, one vehicle working with a directional-forwarding scheme may choose any vehicle within its transmission range as the next-hop forwarder, especially when other vehicle's mobility history or drivers' behaviors are not known. Hence, $\operatorname{Pr}\{\mathrm{R}$ is the $k-$ th node $\mid N=n\}=1 / n$. With this assumption, the analytical results obtained in this letter could be treated as a baseline for comparing or validating more specific network protocols. By substituting the above results into (2), the PDF of $D_{\mathrm{S}}$ can be obtained. Due to the complexity of integral calculations in (4), numerical methods are needed in the derivation process of $f_{D_{\mathrm{S}}}(x)$.

\section{B. Interference Propagation Distance}

Back to the interfering situation depicted in Fig. 1, the notation $D_{\mathrm{B}}$ (or $D_{\mathrm{B}}^{\prime}$ ) is used to represent the distance between the right (or left) boundary of T's ER and the first network node within the right (or left) shaded region. If we assume that the interferer $I$ is the $l$-th node within the right shaded region, then its interference propagation distance $D_{\mathrm{I}}$ can be presented as $D_{\mathrm{I}}=r_{\mathrm{E}}-D_{\mathrm{S}}+D_{\mathrm{B}}+Y_{l-1}$. Since the ER's boundary could be located between two neighboring nodes uniformly, the random variable $D_{\mathrm{B}}$ can be treated as the multiplication of inter-vehicle distance $D$ and another random variable $U$, which is uniformly distributed within $[0,1]$, as $D_{\mathrm{B}}=D \cdot U$. Hence, the CDF of $D_{\mathrm{B}}$ can be directly calculated as

$F_{D_{\mathrm{B}}}(x)=x \cdot \exp \left(\frac{\sigma^{2}}{2}-\mu\right) \cdot \Phi\left(\frac{\mu-\sigma^{2}-\ln x}{\sigma}\right)+\Phi\left(\frac{\ln x-\mu}{\sigma}\right)$.

With $D_{\mathrm{B}}$ 's CDF, its PDF can also be easily obtained.

In view of the independence of $D_{\mathrm{B}}$ and $l$, if we let $Z=D_{\mathrm{B}}+$ $Y_{l-1}, Z$ 's PDF can be derived by the convolution theorem as $f_{Z}(x)=\int_{0}^{\infty} f_{D_{\mathrm{B}}}(t) f_{Y_{l-1}}(x-t)$. Hence, given $D_{\mathrm{S}}=d_{\mathrm{S}}$ and $l, D_{\mathrm{I}}$ 's conditional PDF is a shift of $f_{Z}(x)$ as $f_{D_{\mathrm{I}} \mid\left(d_{\mathrm{S}}, l\right)}(x)=f_{Z}\left(x-r_{\mathrm{E}}+d_{\mathrm{S}}\right)$. 
To remove the condition on $l$, the total probability formula can be applied again as

$$
f_{D_{\mathrm{I}} \mid d_{\mathrm{S}}}(x)=\sum_{l=1}^{\left\lfloor r_{\mathrm{E}} / L_{\mathrm{V}}\right\rfloor} f_{D_{\mathrm{I}} \mid\left(d_{\mathrm{S}}, l\right)}(x) \cdot p_{l},
$$

where $p_{l}$ is the probability that the interferer $I$ is the $l$-th node within the right shaded region. Similarly to (3), by using $N_{\mathrm{r}}$ to represent the total number of nodes in the right shaded region, $p_{l}$ could be expanded as

$$
p_{l}=\sum_{n_{\mathrm{r}}=1}^{\left\lfloor r_{\mathrm{E}} / L_{\mathrm{v}}\right\rfloor} \operatorname{Pr}\left\{N_{\mathrm{r}}=n_{\mathrm{r}}\right\} \operatorname{Pr}\left\{I \text { is the } l \text {-th } \mid N_{\mathrm{r}}=n_{\mathrm{r}}\right\} .
$$

The first part within the summation of (7) can be obtained with the identical method used in (4). Due to the space limit, the detailed derivation process is omitted here. Meanwhile, if given each node's average transmission probability $p_{\mathrm{t}}$, the remaining conditional probability in (7) can be presented as ${ }^{3}$

$$
\operatorname{Pr}\left\{I \text { is the } l \text {-th } \mid N_{\mathrm{r}}=n_{\mathrm{r}}\right\}=\frac{p_{\mathrm{t}}\left(1-p_{\mathrm{t}}\right)^{n_{\mathrm{r}}-1}}{n_{\mathrm{r}}} .
$$

Note that, due to the homogenous assumption of network nodes' transmission probability, the conditional probability calculated in (13) is independent with $l$, which is similar to the idea presented in (7). The calculation of $p_{\mathrm{t}}$ is beyond the scope of this letter, moreover, it has already been investigated for the VANETs scenario (e.g., [9]). With all these above results, $f_{D_{\mathrm{I}} \mid d_{\mathrm{S}}}(x)$ can be obtained, and by applying the similar method, the conditional PDF $f_{D_{I}^{\prime} \mid d_{S}}(x)$ for the propagation distance of the left-side interferer $I^{\prime}$ can also be derived.

\section{Capacity Characteristics}

Given $f_{D_{\mathrm{I}} \mid d_{\mathrm{S}}}(x)$ and the propagation model described in Section II, the CDF of I's interference power $P_{\mathrm{I}}$ received at R could be presented as

$$
\begin{aligned}
F_{P_{\mathrm{I}} \mid d_{\mathrm{S}}}(x) & =\operatorname{Pr}\left\{P_{\mathrm{I}}<x \mid D_{\mathrm{S}}=d_{\mathrm{S}}\right\} \\
& =\int_{t^{\prime}=0}^{x} \int_{t=0}^{2 r_{\mathrm{E}}} f_{P_{\mathrm{r}} \mid d_{\mathrm{I}}}\left(t^{\prime}\right) \cdot f_{D_{\mathrm{I}} \mid d_{\mathrm{S}}}(t) \mathrm{d} t \mathrm{~d} t^{\prime}
\end{aligned}
$$

and the CDF of $I^{\prime}$ 's interference power at R can also be derived as $F_{P_{I}^{\prime}} \mid d_{S}(x)$, which could be easily transferred to their PDF $f_{P_{\mathrm{I}} \mid d_{\mathrm{S}}}(x)$ and $f_{P_{\mathrm{I}}^{\prime} \mid d_{\mathrm{S}}}(x)$, respectively. Hence, the total interference power accumulated at the receiver $\mathrm{R}$ is the sum of two independent random variables, as

$$
f_{P_{\mathrm{I}}+\mathrm{I}^{\prime} \mid d_{\mathrm{S}}}(x)=\int_{0}^{\infty} f_{P_{\mathrm{I}} \mid d_{\mathrm{S}}}(x-t) \cdot f_{P_{\mathrm{I}}^{\prime} \mid d_{\mathrm{S}}}(t) \mathrm{d} t .
$$

Given $D_{\mathrm{S}}=d_{\mathrm{S}}$, the SIR at $\mathrm{R}$ is the ratio of two random variables, and then its conditional PDF could be presented as

$$
f_{\mathrm{SIR} \mid d_{\mathrm{S}}}(x)=\int_{0}^{\infty} t \cdot f_{P_{\mathrm{r}} \mid d_{\mathrm{S}}}(t \cdot x) \cdot f_{P_{\mathrm{I}}+\mathrm{I}^{\prime} \mid d_{\mathrm{S}}}(t) \mathrm{d} t .
$$

\footnotetext{
${ }^{3}$ Strictly speaking, the transmission probability for each node should be different. For example, the nodes in the shaded region with a shorter distance to $\mathrm{T}$ should have a higher probability to access the channel successfully, as more of its neighbors are depressed by $\mathrm{T}$. This issue will not be discussed in this letter, but will be investigated in our future work.
}

Finally, the SIR's PDF can be derived as

$$
f_{\mathrm{SIR}}(x)=\int_{0}^{r_{\mathrm{E}}} f_{\mathrm{SIR} \mid d_{\mathrm{S}}}(x) \cdot f_{D_{\mathrm{S}}}(t) \mathrm{d} t, x>0
$$

and then the CDF of a randomly selected communication pair's link capacity $C$ is

$$
F_{C}(x)=\operatorname{Pr}\left\{W \log _{2}(1+\operatorname{SIR})<x\right\}=F_{\text {SIR }}\left(2^{\frac{x}{W}-1}\right)
$$

where $W$ is the bandwidth allocated to the observed communication pair, therefore, the random variable $C$ 's PDF could be given as $f_{C}(x)=\frac{\ln 2}{W} \cdot 2^{\frac{x}{W}-1} f_{\mathrm{SIR}}\left(2^{\frac{x}{W}-1}\right)$.

With $f_{C}(c)$, it is easy to calculate the expectation of a randomly selected communicating link's capacity $E(C)$. Moreover, it is interesting to find out that, when the node density is high, the ER arrangement in VANETs is identical to the classic Rényi's packing problem [10], [11], which focused on how many segments with identical length can be put in an interval under the constraint that the centers of these segments cannot be covered by another segment. Based on the results of [10], the expected number of concurrent transmitting node pairs on a line segment with length $L_{\mathrm{N}}$ can be estimated as $\gamma L_{\mathrm{N}} /\left(2 r_{\mathrm{E}}\right)$ ( $\gamma$ is a constant with approximate value 1.4952), when $L_{\mathrm{N}}$ is relatively large. Hence, the expected network capacity for a VANET deployed within a road length $L_{\mathrm{N}}$ could be obtained by $E_{\mathrm{N}}(C)=\gamma L_{\mathrm{N}} E(C) /\left(2 r_{\mathrm{E}}\right)$.

\section{Numerical and Simulation Results}

To verify the analytical results, a series of simulations has been conducted with Matlab. The Nakagami fading model is utilized with different fading factors, and the value of pathloss exponent $\alpha$ is selected by referring to a vehicular communication-based field test result [12] as 3.18. Meanwhile, according to the IEEE 802.11 standard, the CCA threshold $P_{\mathrm{th}}$ is set to $-60 \mathrm{dBm}$, and the core parameters for the MAC scheme are set as: slot time duration $13 \mu \mathrm{s}$, SIFS duration $32 \mu \mathrm{s}$, minimum contention window $(\mathrm{CW})$ size $\mathrm{CW}_{\min }=15$, and maximum $\mathrm{CW}$ size $\mathrm{CW}_{\max }=1023$. For different simulation scenarios, the vehicular transmission power varies from $200 \mathrm{~mW}$ to $600 \mathrm{~mW}$, which matches with the common characteristics of mobile stations, and the expected safe distance between moving vehicle is chosen from $100 \mathrm{~m}, 200 \mathrm{~m}$, and $300 \mathrm{~m},{ }^{4}$ which leads to different values of $\mu$ in the Car-Following model as 4.602, 6.764, and 9.352, and the corresponding $\sigma$ are 0.080 , 0.115 , and 0.363 , respectively. Moreover, to obtain enough data to illustrate smooth curves, each parameter combination is simulated by the Monte Carlo method for $10^{8}$ times.

In Fig. 2, the conditional PDF of SIR at the tagged receiver is illustrated with different values of the signal propagation distance $d_{\mathrm{S}}$ and the fading factor $m$, while the ratio $\gamma=r_{\mathrm{T}} / r_{\mathrm{E}}$ is kept as 1 . Generally, when the signal propagation distance is increased, the probability for a receiver to obtain a high SIR is decreased, which is mainly due to the obvious decrease of the received signal power. Moreover, when $d_{\mathrm{S}}=300 \mathrm{~m}$, the analytical results for an extreme interfering case, in which the two interferers are the very first nodes outside the tagged transmitter's ER, are also illustrated. Comparing with the extreme

\footnotetext{
${ }^{4}$ The required safe distance might be different for different counties or regions, so here we use the common 100-meter requirement in China as the baseline and slightly increase it to a relatively large value.
} 


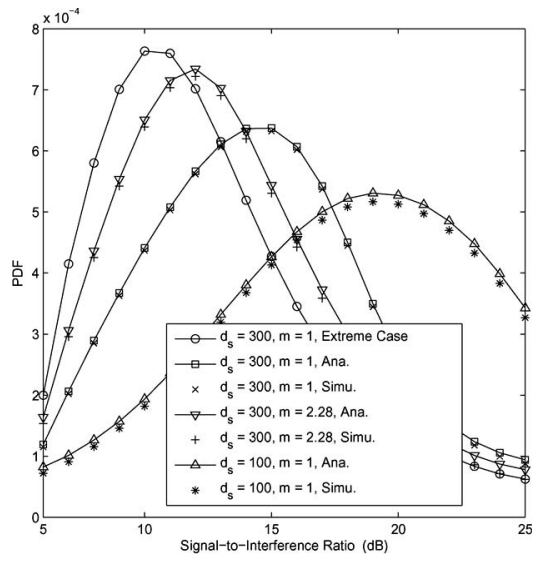

Fig. 2. Conditional PDF of SIR at receiver, $\mu=4.602, \sigma=0.080, P_{\mathrm{t}}=0.4 \mathrm{~W}$, $\gamma=1$.

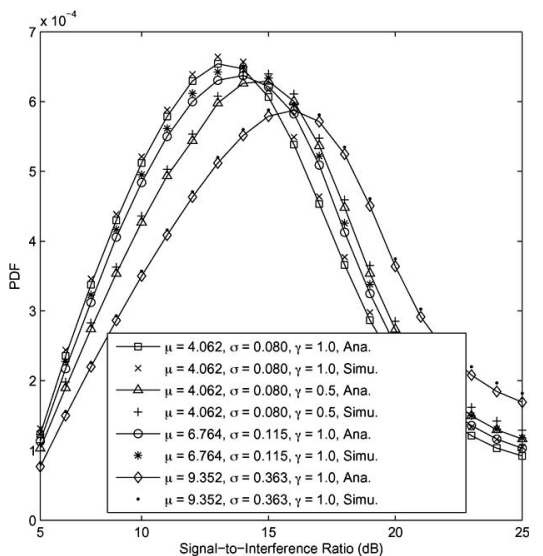

Fig. 3. Impact of mobility pattern on SIR, $d_{\mathrm{s}}=200 \mathrm{~m}, P_{\mathrm{t}}=0.4 \mathrm{~W}$.

case, the general interfering situation demonstrates a higher probability to finish a high-quality data exchange. When $m$ is increased from 1, which represents the Rayleigh fading, to 2.28, which equals to a Rician fading with its $K$ factor as 3 , the peak point of the conditional SIR curve is moved to a lower SIR region, which matches with the differences between the impact of Rayleigh and Rician fading model.

For demonstrating the impact of the safe driving distance on the communication quality, the SIR's conditional PDF is depicted with different $\mu-\sigma$ combinations and $\gamma$ in Fig. 3. As described earlier, larger $\mu$ represents a longer safe driving distance, which directly affects $D_{\mathrm{B}}$. Therefore, Fig. 3 illustrates an opposite changing pattern to Fig. 2, as the peak point for the PDF curve is shifted to the high SIR region with the increase of $\mu$. On the other hand, the increase of $\mu$ has a much smaller impact on SIR comparing with $d_{\mathrm{S}}$ in the previous simulation group. Therefore, with the reasonable settings of the expected safe distance, e.g., required by the traffic regulations, the impact generated by the small-scale changing of the mobility pattern can safely be ignored. Moreover, when $\gamma$ is decreased from 1 to 0.5 , the transmission quality is also improved. This is mainly due to the fact that, the reduced transmission range decreases the chance for a longer signal propagation distance.

Finally, the average network capacity is illustrated in Fig. 4 with the changed transmission power and road segment's length. Although a lower transmission power may directly decrease the SIR at each receiver, the number of concurrent communicating pairs in a given road segment can also be greatly increased. According to the figure, the number of the

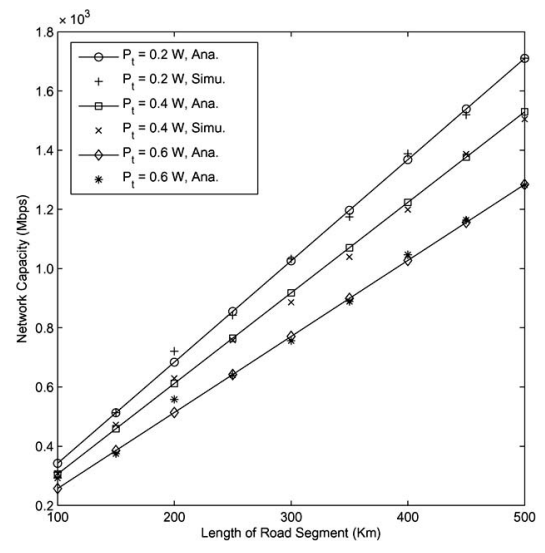

Fig. 4. Network capacity with changed $L_{\mathrm{v}}$ and $P_{\mathrm{t}}$, while $\mu=4.602, \sigma=0.080$, $\gamma=1, m=1$.

concurrent pairs will dominate network capacity's changing pattern. Moreover, it is also clear that, the increasing rate of the network capacity is decreased with $P_{\mathrm{t}}$ 's increasing, which means that, when the interference level is acceptable, the VANETs should use a relatively lower power to improve the overall network performance.

\section{CONCLUSION}

In this letter, we analyzed the interference-based capacity of VANETs based on the classic Car-Following model. With the similar analysis method, the VANETs capacity of the multilane scenario can be studied by considering the effect of lanechanging on the stochastic property of inter-vehicle distances. Moreover, by utlizing the existing results on heterogeneous transmission probability in VANETs, more realistic results for VANETs capacity can also be obtained. These will be the follow-on work for this letter in the near future.

\section{REFERENCES}

[1] M. Wang et al., "Asymptotic throughput capacity analysis of VANETs exploiting mobility diversity," IEEE Trans. Veh. Technol., doi: 10.1109/ TVT.2014.2363791, to be published.

[2] N. Lu and X. Shen, Capacity Analysis of Vehicular Communication Networks. New York, NY, USA: Springer-Verlag, 2013.

[3] K. Hafeez, L. Zhao, B. Ma, and J. Mark, "Performance analysis and enhancement of the DSRC for VANET's safety applications," IEEE Trans. Veh. Technol., vol. 62, no. 7, pp. 3069-3083, Sep. 2013.

[4] I. Greenberg, "The log-normal distribution of headways," Australian Road Res., vol. 2, no. 7, pp. 14-18, 1966.

[5] P. Gupta and P. Kumar, "The capacity of wireless networks," IEEE Trans. Inf. Theory, vol. 46, no. 2, pp. 388-404, Mar. 2000.

[6] C. Campolo, A. Molinaro, and A. Vinel, "Understanding the performance of short-lived control broadcast packets in 802.11 p/wave vehicular networks," in Proc. IEEE VNC, 2011, pp. 102-108.

[7] T. Yang, G. Mao, and W. Zhang, "Connectivity of large-scale CSMA networks," IEEE Trans. Wireless Commun., vol. 11, no. 6, pp. 2266-2275, Jun. 2012.

[8] L. Fenton, "The sum of log-normal probability distributions in scattered transmission systems," IRE Trans. Commun. Syst., vol. 8, no. 1, pp. 5767, Mar. 1960.

[9] X. Ma and X. Chen, "Performance analysis of IEEE 802.11 broadcast scheme in Ad Hoc wireless LANs," IEEE Trans. Veh. Technol., vol. 57, no. 6, pp. 3757-3768, Nov. 2008.

[10] A. Rényi, "On a one-dimensional problem concerning random spacefilling," Publ. Math. Inst. Hung. Acad. Sci., vol. 3, no. 1/2, pp. 109-127, 1958.

[11] A. Giang, A. Busson, D. Gruyer, and A. Lambert, "A packing model to estimate VANET capacity," in Proc. IEEE IWCMC, 2012, pp. 1119-1124.

[12] O. Altintas et al., "Field tests and indoor emulation of distributed autonomous multi-hop vehicle-to-vehicle communications over TV white space," in Proc. ACM Mobicom, 2012, pp. 439-442. 